\title{
Transonic similarity solution for aligned field MHD nozzle flow
}

\author{
H. WATANABE, M. SICHEL AND R. S. B. ONG \\ Department of Aerospace Engineering, The University of Michigan, Ann Arbor, Michigan, U.S.A. \\ (Received April 18, 1972)
}

SUMMARY

The transonic flow near the throat of a converging-diverging nozzle of a gas with infinite electrical conductivity is considered. The magnetic field $\mathbf{B}$ is everywhere aligned with the velocity $\mathbf{q}$ so that the equations describing the flow are reducible to those of ordinary gasdynamics. Thus, it is possible to utilize the transonic similarity solution of Tomotika and Tamada [3] to study aligned field magnetohydrodynamic flow near a nozzle throat. Only transonic flows are considered, and the structures of sub- and supersonic flows with speeds greater and less than the Alfvén speed are investigated.

\section{Introduction}

Flow through a converging-diverging nozzle is one of the classical problems of gas dynamics. As the exit pressure is reduced the nozzle first behaves like a venturi with the maximum velocity at the throat. As the exit pressure continues to decrease pockets of supersonic flow develop near the walls of the nozzle throat where the flow thus becomes transonic. This type of flow was first investigated by Taylor [1] and is sometimes called Taylor flow. Here we investigate such transonic flows for a gas with infinite conductivity and with the magnetic induction B everywhere parallel to or aligned with, the gas velocity q. As indicated by Grad [2] the equations of such "aligned field" magnetohydrodynamic (MHD), flows can always be reduced to the equations of ordinary gas dynamics. We have used this property and the well known similarity solutions of Tomotika and Tamada [3] for transonic nozzle flow to study the structure of such MHD Taylor flows.

As the exit pressure is reduced further a transition occurs from the Taylor flow which is symmetrical with respect to the throat to a continuously accelerating flow, subsonic upstream, and supersonic downstream of the throat. Such flows were first studied by Meyer [4] and, hence are sometimes called "Meyer" flows. The Tomotika-Tamada solution also describes the transonic portion of such Meyer flows; however, the transition from the Taylor to the Meyer type flow involves the formation of shock waves within the nozzle, and so, as shown by Sichel [5], cannot be described by a purely inviscid theory.

Aligned field MHD flows are complicated by the existence of three critical speeds; the speed of sound a, the Alfvén speed $b$, and the speed $b a /\left(b^{2}+a^{2}\right)^{\frac{1}{2}}$. With a sufficiently strong magnetic field accelerating flow in a converging-diverging nozzle thus passes through three transitions as the velocity $\mathbf{q}$ increases for the equations describing the flow are elliptic when $|\mathbf{q}|<b a$ / $\left(b^{2}+a^{2}\right)^{\frac{1}{2}}$, are hyperbolic for $b a /\left(b^{2}+a^{2}\right)^{\frac{1}{2}}<|\mathbf{q}|<a$, are elliptic when $a<|\mathbf{q}|<b$, and finally become hyperbolic once more when $|\mathbf{q}|>b$. In neutral gas flow the only transition occurs at $|\mathbf{q}|=a$. Series solutions of Meyer type aligned field flows with three transitions have been investigated by Chu [6] who examined the character of the flow near each of the above transitions; however, he did not consider the symmetrical Taylor type flow.

Only the transonic portion of the nozzle is considered in the present paper. The equations and properties of aligned field flow are first presented. Then the Tomotika-Tamada solution is described and used to investigate the structure of transonic Taylor and Meyer aligned field MHD flow. 


\section{Formulation}

When the velocity vector $\mathbf{q}$ and the magnetic field $\mathbf{B}$ of an inviscid gas with infinite conductivity are parallel so that

$$
\lambda \mathbf{B}=\rho \mathbf{q}
$$

the Lundquist equations of MHD can be reduced to the two equations [2]:

$$
\begin{aligned}
& \operatorname{div}(\rho \mathbf{q})=0 \\
& \operatorname{curl}\left[\left(1-A^{-2}\right) \mathbf{q}\right]=0 .
\end{aligned}
$$

In (3) the Alfvén number $A=q / b$, and $b$ is the Alfvén wave velocity given by $b=B /(\mu \rho)^{\frac{1}{2}}$ with $\mu$ the magnetic permeability in a vacuum. In a flow starting from uniform conditions $\lambda$ is constant throughout the flow. The flow is isentropic and since the electromagnetic body force $\mathbf{j} \times \mathbf{B}$ only can act normal to the streamtube direction the stagnation enthalpy remains constant so that

$$
h+\frac{q^{2}}{2}=h_{0}=\text { const } .
$$

In the limit of vanishing magnetic field $A \rightarrow \infty$ and Eqs. (2) and (3) reduce to the equations for irrotational compressible flow.

In natural coordinates with $S$ parallel to, and $r$ perpendicular to the streamlines the aligned field flow equations (2) and (3) become

$$
\begin{aligned}
& \left(1-A^{2}\right) q \frac{\partial \theta}{\partial S}+\left(M^{2}+A^{2}-1\right) \frac{\partial \theta}{\partial r}=0 \\
& \left(1-M^{2}\right) \frac{\partial q}{\partial S}+q \frac{\partial \theta}{\partial r}=0
\end{aligned}
$$

with $q$ and $\theta$ the magnitude and direction of the velocity and with $M$ the Mach number. The characteristics of (5) and (6) are real in the regions

and

$$
A<1, \quad M<1, \quad M^{2}+A^{2}>1
$$

$$
A>1, \quad M>1
$$

where the equations are hyperbolic, and (5) and (6) are elliptic in the regions

and

$$
\begin{array}{ll}
M<1, & A>1 \\
M>1, & A<1
\end{array}
$$

$$
M^{2}+A^{2}<1
$$

These regions are shown on the $M-A$ plane, or Resler diagram in Fig. 1. The isentropes sketched in Fig. 1 show that an accelerating flow may pass through three transitions at $M^{2}+A^{2}=1$, $M=1$ and $A=1$, and as already mentioned such flows have been studied by Chu [6]. Equations (5) and (6) show that for finite $\partial q / \partial S, \partial \theta / \partial r=0$ at the sonic transition, $M=1$, i.e. the streamtube has a throat. At the Alfvén transition $\partial q / \partial r=0$, i.e. the electromagnetic and centrifugal forces balance so that there is no velocity or pressure variation normal to the streamlines. At $M^{2}+A^{2}=1$, which is called the hypercritical transition, $\partial \theta / \partial S=0$, so that the streamline has an inflection point. Here the pressure and electromagnetic forces balance causing the streamline curvature to vanish.

The present paper deals with sub-Alfvénic and super-Alfvénic transonic flow near the throat of a nozzle, and the appropriate regimes are indicated in Fig. 1. For two-dimensional perfect gas flow perturbed from sonic conditions, Kogan [7], Seebass [8], and others have shown that expanding the velocity and stretching the coordinates according to 


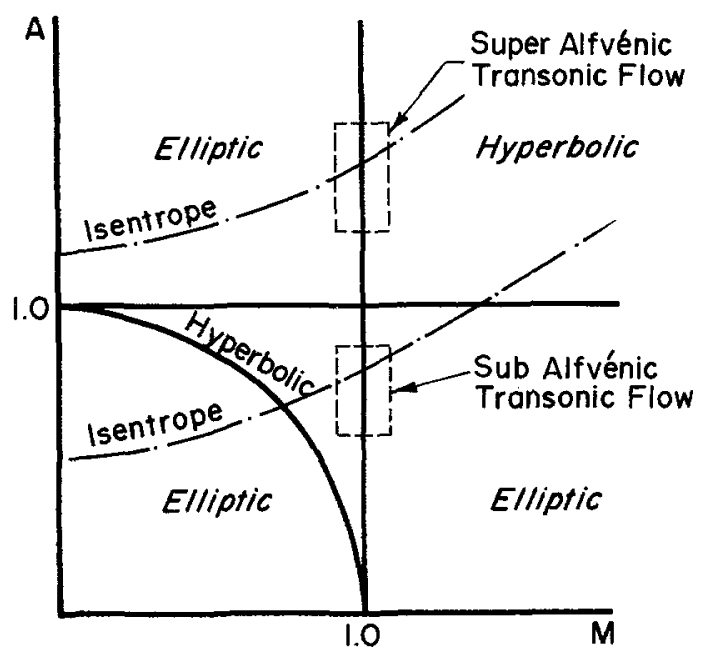

Figure 1. The $M-A$ plane or Resler diagram.

$$
\begin{aligned}
& \frac{\bar{u}}{\bar{a}^{*}}=1+\varepsilon \tilde{u}+\ldots ; \quad \frac{\bar{v}}{\bar{a}^{*}}=\varepsilon^{\frac{3}{2}} \tilde{v}+\ldots \\
& y=\frac{\bar{y}}{\bar{h}} ; \quad x=\frac{\bar{x}}{\bar{h} \varepsilon^{\frac{1}{2}}}
\end{aligned}
$$

leads to the perturbation equations

$$
\begin{aligned}
& -(\gamma+1) \tilde{u} \frac{\partial \tilde{u}}{\partial x}+\frac{\partial \tilde{v}}{\partial y}=0 \\
& \left(1-A_{*}^{-2}\right) \frac{\partial \tilde{v}}{\partial x}-\frac{\partial \tilde{u}}{\partial y}=0
\end{aligned}
$$

valid for transonic aligned field MHD flow provided $A_{*} \neq 1$. The asterisk denotes quantities evaluated at the sonic velocity. In (7) barred quantities are dimensional, $\varepsilon$ is a small parameter related to the deviation of the flow from the sonic or critical speed $\bar{a}^{*}, \bar{h}$ is a characteristic dimension (the nozzle half height in the present case) and $A_{*}$ is the Alfvén number evaluated at critical or sonic conditions.

To the order of the expansion (7) the velocity magnitude $|\mathbf{q}|$ is given by

$$
|\mathbf{q}|=\left[\bar{u}^{2}+\bar{v}^{2}\right]^{\frac{1}{2}}=a^{*}\left[1+\varepsilon \tilde{u}+O\left(\varepsilon^{2}\right)\right]
$$

so that the flow is supersonic when $\tilde{u}>0$ and subsonic with $\tilde{u}<0$. The equation for the characteristics of $(8)$ and $(9)$ are readily shown to be

$$
\left(\frac{\mathrm{d} y}{\mathrm{~d} x}\right)_{c}= \pm\left[(\gamma+1) \tilde{u}\left(1-A_{*}^{-2}\right)\right]^{\frac{1}{2}}
$$

Equation (11) now shows that in super-Alfvénic flow when $A_{*}>1$ or $A_{*}^{-2}<1$ the characteristics are real and the flow is hyperbolic in supersonic flow when $\tilde{u}>0$, as in neutral gas flow. In subAlfvénic flow, $A_{*}>1$, and the flow is hyperbolic in subsonic flow when $\tilde{u}<0$. The behavior of the perturbation equations (8) and (9) is thus in accord with the Resler diagram (Fig. 1). With the transformation

$$
\begin{aligned}
& \tilde{u}=\left(1-A_{*}^{-2}\right) U, \quad \tilde{v}=(\gamma+1)^{\frac{1}{2}}\left(1-A_{*}^{-2}\right) V \\
& y=Y, \quad x=(\gamma+1)^{\frac{1}{2}}\left(1-A_{*}^{-2}\right) X
\end{aligned}
$$

Equations (8) and (9) become 


$$
\begin{array}{r}
-U \frac{\partial U}{\partial X}+\frac{\partial V}{\partial Y}=0 \\
\frac{\partial V}{\partial X}-\frac{\partial U}{\partial Y}=0
\end{array}
$$

Equations (13).and (14) are a form of the transonic small disturbance equations of ordinary gas dynamics, and this reduction of the aligned field equations (8) and (9) has already been indicated by Grad [2]. Upon eliminating $V$, Eq. (13) and (14) can be reduced to the single equation

$$
-\frac{\partial^{2}}{\partial X^{2}}\left(\frac{U^{2}}{2}\right)+\frac{\partial^{2} U}{\partial Y^{2}}=0
$$

\section{The similarity solution}

Tomotika and Tamada [3] showed that the transformation

$$
\begin{aligned}
& \frac{1}{2} U=Z(S)+2 Y^{2} \\
& S=X+Y^{2}
\end{aligned}
$$

reduces the transonic equation (15) to the ordinary differential equation

$$
Z Z^{\prime \prime}+\left(Z^{\prime}-2\right)\left(Z^{\prime}+1\right)=0
$$

with the solution

$$
(Z-2 S)^{2}(Z+S)=2 \alpha^{3}
$$

The form of the solution (18) is determined by the arbitrary parameter $\alpha$. The flow described by transformation (16) is symmetrical about the $X$-axis and so may be considered as the flow through a nozzle, since any streamline can be considered as the nozzle wall. The function $2 Z(S)$ corresponds to the variation of velocity $U$ along the nozzle axis with $Z<0$ and $Z>0$ corresponding to subsonic and supersonic flow respectively. The dimensionless velocity $V$ determined from the irrotationality condition (14) and equation (17) is given by

$$
V=4\left[Y Z(S)+2 X Y+\frac{2}{3} Y^{3}\right] \text {. }
$$

In the special case $\alpha=0$ Eq. (18) shows that $Z$ has the two solutions

$$
\begin{aligned}
& Z=2 S \\
& Z=-S
\end{aligned}
$$

the first of which corresponds to continuously accelerating or "Meyer" flow, and the second corresponds to continuously decelerating flow. When $\alpha \neq 0$ determination of $Z$ from (18) in explicit form requires solution of a cubic equation with the result:

$$
\begin{array}{ll}
Z=\left(\eta+\sqrt{ } \alpha^{3} \xi\right)^{\frac{1}{3}}+\left(\eta-\sqrt{\left.\alpha^{3} \xi\right)^{\frac{1}{3}}+S ;}\right. & \alpha^{3} \xi>0 \\
Z=2|S| \cos \left(\frac{1}{3} \tan ^{-1} \frac{\sqrt{ }-\alpha^{3} \xi}{\eta}+\frac{2}{3} \pi\right)+S ; & \alpha^{3} \xi<0, \eta>0 \\
Z=2|S| \cos \left(\frac{1}{3} \tan ^{-1} \frac{\sqrt{ }-\alpha^{3} \xi}{\eta}+\pi\right)+S ; & \alpha^{3} \xi<0, \eta<0
\end{array}
$$

where $\xi=\alpha^{3}-2 S^{3}$ and $\eta=\alpha^{3}-S^{3}$.

The variation of $Z(S)$ is shown in Fig. 2 for several different values of $\alpha$. The solutions in regions $A^{\prime}$ and $B$ represent physically unrealizable flows since the acceleration $Z^{\prime}$ becomes infinite where $Z=0$. The solutions in region $A$ correspond to Taylor flow with subsonic velocities both upstream and downstream of the nozzle throat. Region $B^{\prime}$ describes flow which is supersonic upstream and downstream of the throat, and which, at least for a neutral gas, can 


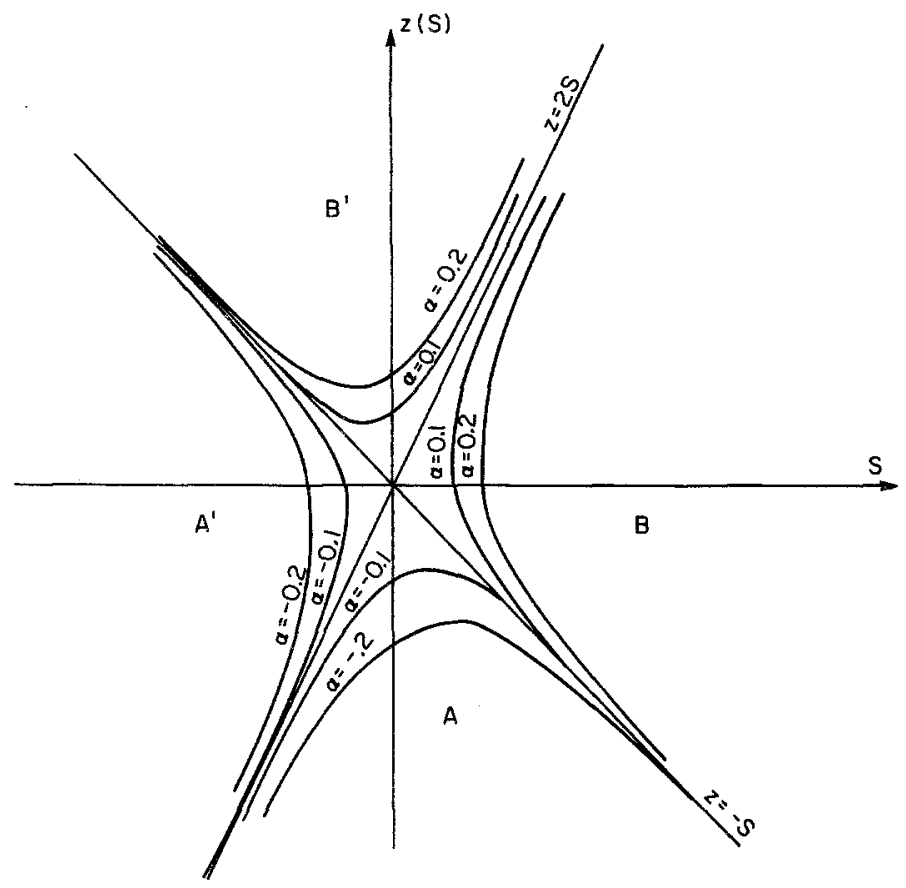

Figure 2. The Tomotika-Tamada nozzle similarity solution.

be shown to be unstable. In the limit $\alpha \rightarrow 0$ branch $A$ approaches the limiting Taylor, or symmetric solution

$$
\begin{array}{ll}
Z=2 S, & S<0 \\
Z=-S, & S>0
\end{array}
$$

in which the velocity on the nozzle axis just attains the sonic value. The limiting solution for branch $B^{\prime}$ is

$$
\begin{array}{ll}
Z=-S, & S<0 \\
Z=2 S, & S>0 .
\end{array}
$$

Significantly, the Taylor solution does not approach the Meyer solution $Z=2 S$ in the limit $\alpha \rightarrow 0$, i.e. the transition from the Taylor to Meyer flow cannot be described within the framework of the purely inviscid theory but requires the introduction of viscous terms or shock discontinuities [5]. Nevertheless, many aspects of the structure of transonic nozzle flow can be ascertained by a detailed study of the Taylor, Meyer, and limiting solutions [3]. Through the transformation (12) the Tomotika-Tamada solution will now be used to study aligned field transonic nozzle flow.

\section{Continuously accelerating and decelerating flows}

We now consider the "Meyer" flow corresponding to $Z=2 S$ in detail. From the definition of $S$ and the transformation (12) the perturbation velocities $\tilde{u}$ and $\tilde{v}$ are found to be

$$
\begin{aligned}
& \tilde{u}=4 x(\gamma+1)^{-\frac{1}{2}}+8\left(1-A_{*}^{-2}\right) y^{2} \\
& \tilde{v}=16 x y+\left(\frac{32}{3}\right)(\gamma+1)^{\frac{1}{2}}\left(1-A_{*}^{-2}\right) y^{3}
\end{aligned}
$$

Equation (25) shows that only the variation of $\tilde{u}$ with $y$ is affected by the magnetic field strength or $A_{*}^{-2}$; the velocity $\tilde{u}(x, 0)$ along the nozzle axis is independent of $A_{*}$. Thus $\tilde{u}$, which, to the present order of approximation, also corresponds to the fluid speed increases with $y$ for superAlfvénic flow with $A_{*}^{-2}<1$ and decreases with $y$ for sub-Alfvénic flow with $A_{*}^{-2}>1$. Consequently lines of constant speed or isovels will as in electrically neutral flow be concave up- 
stream for super-Alfvénic flow but will be conves upstream for sub-Alfvénic flow. This result is in accord with the discussion in Section 2 , and reflects the fact that with increasing field strength the electromagnetic body force at first provides some of the centrifugal force needed to balance the streamline curvature. Once $A_{*}^{-2}>1$ the electromagnetic force exceeds the centrifugal acceleration and so is in part balanced by the pressure which thus increases with increasing $y$ rather than decreasing as in neutral or super-Alfvénic flow. Typical constant velocity contours or isovels for Meyer flow are shown qualitatively in Fig. 3(a) and 3(b), which are part of a map of the various aligned field nozzle flows corresponding to the Tomotika-Tamada solution. The structure of nozzle flow which is decelerating from supersonic to subsonic flow, corresponding to $Z=-S$ is shown in Fig. 3(c) and 3(d). In the neutral case such flows are unstable and always are accompanied by the formation of shock waves.

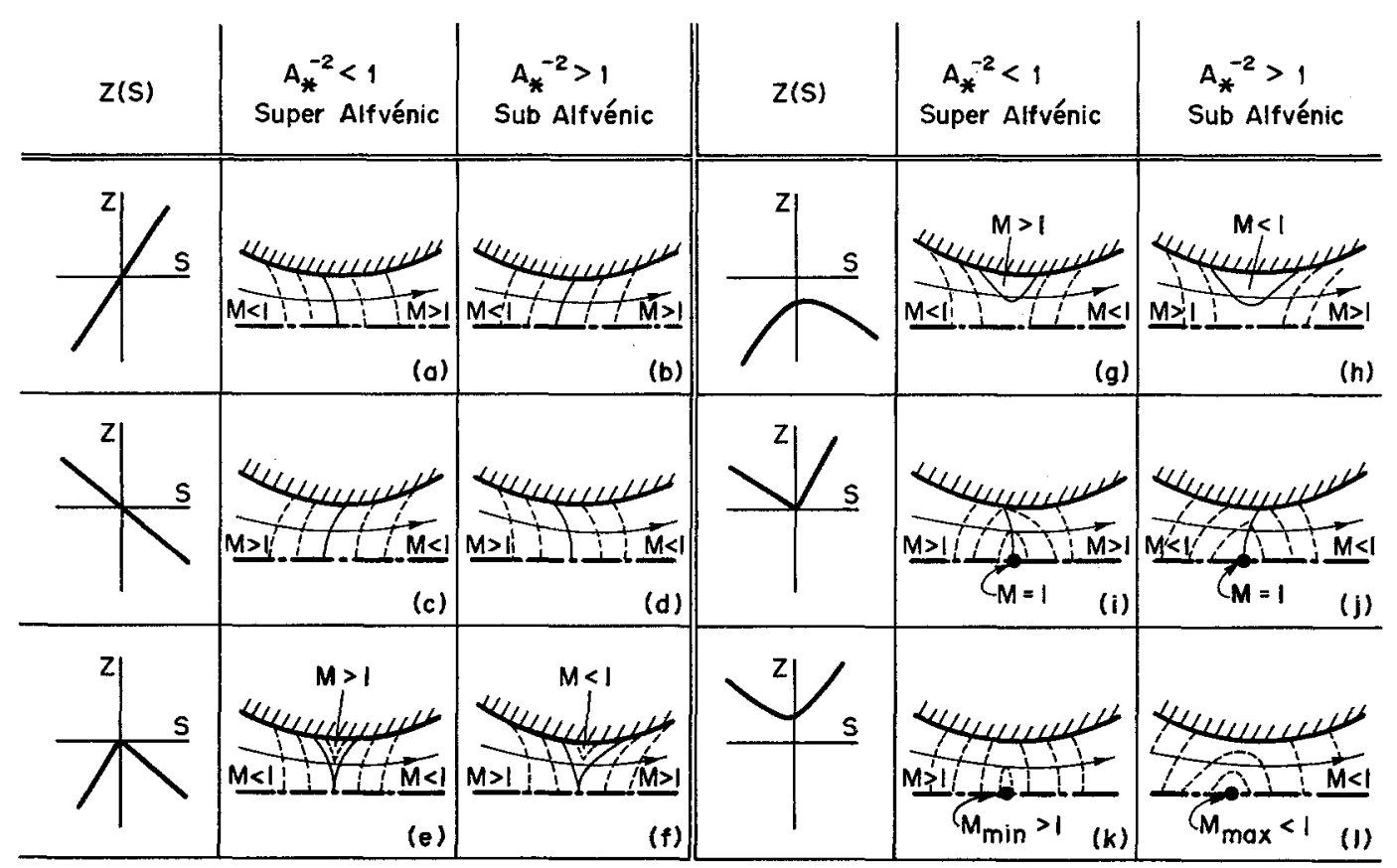

Figure 3. Aligned field nozzle flows corresponding to the Tomotika-Tamada solution.

The streamlines of the flow can be determined by solving the differential equation

$$
\frac{d \bar{y}}{d \bar{x}}=\frac{\bar{v}}{\bar{u}}=\frac{\varepsilon^{\frac{3}{2}} \tilde{v}}{1+\varepsilon \tilde{u}}
$$

which in terms of $x$ and $y$ as defined by (7) becomes

$$
\frac{d y}{d x}=\varepsilon^{2} \tilde{v}+O\left(\varepsilon^{3}\right)
$$

One of these streamlines can be chosen as the nozzle wall and the location of the nozzle throat is then determined by

$$
\frac{d y}{d x}=0=\varepsilon^{2} \tilde{v}=16 \varepsilon^{2}\left[x y+\frac{2}{3}(y+1)^{\frac{1}{2}}\left(1-A_{*}^{-2}\right) y^{3}\right]
$$

so that

$$
x_{t}=-\frac{2}{3}(\gamma+1)^{\frac{1}{2}}\left(1-A_{*}^{-2}\right) y_{t}^{2}
$$

and choosing the characteristic length $\bar{h}$ the nozzle half height, $y_{t}=1.0$. If the integration of (27) 
is started at the throat then $\eta=(y-1)$, the deviation of the wall streamline from the throat value, will be $O\left(\varepsilon^{2}\right)$ from (27) so that the equation of the wall streamline can be written in the form

$$
\frac{d \eta}{d x}=16 \varepsilon^{2}\left[x+\frac{2}{3}(\gamma+1)^{\frac{1}{2}}\left(1-A_{*}^{-2}\right)\right]+O\left(\varepsilon^{4}\right) .
$$

Integration of (30) then yields

$$
y_{w}=1+\eta=1+16 \varepsilon^{2}\left[\frac{x^{2}-x_{t}^{2}}{2}+\frac{2}{3}(\gamma+1)^{\frac{1}{2}}\left(1-A_{*}^{-2}\right)\left(x-x_{t}\right)\right]
$$

for the ordinate of the nozzle wall.

The wall curvature at the throat where $(d y / d x)=0$ is readily determined from (28) as

$$
\frac{\bar{h}}{\bar{R}}=\frac{1}{\varepsilon}\left(\frac{d^{2} y}{d x^{2}}\right)_{t}=16 \varepsilon
$$

where $\bar{R}$ is the nozzle wall radius of curvature at the throat. Hence the wall curvature at the throat is always positive and independent of the magnetic field strength. For nozzle flow the small parameter $\varepsilon$, which is of the order of the deviation of the velocity from the sonic value, thus depends on the throat radius of curvature.

The characteristics of the flow are the two parabolas

$$
\begin{aligned}
& x / y^{2}=2(\gamma+1)^{\frac{1}{2}}\left(1-A_{*}^{-2}\right) \\
& x / y^{2}=-(\gamma+1)^{\frac{1}{2}}\left(1-A_{*}^{-2}\right)
\end{aligned}
$$

which are obtained by substituting $\tilde{u}$ from (25) in the characteristic equation (11). The sonic line, $\tilde{u}=0$, is also a parabola described by the equation

$$
x / y^{2}=-2(\gamma+1)^{\frac{1}{2}}\left(1-A_{*}^{-2}\right)
$$

and the line of zero streamline slope, $\tilde{v}=0$, is described by

$$
x / y^{2}=-\frac{2}{3}(\gamma+1)^{\frac{1}{2}}\left(1-A_{*}^{-2}\right) .
$$

The characteristics, the sonic line and the zero slope line are shown qualitatively in Fig. 4 for super and sub Alfvénic accelerating nozzle flow.

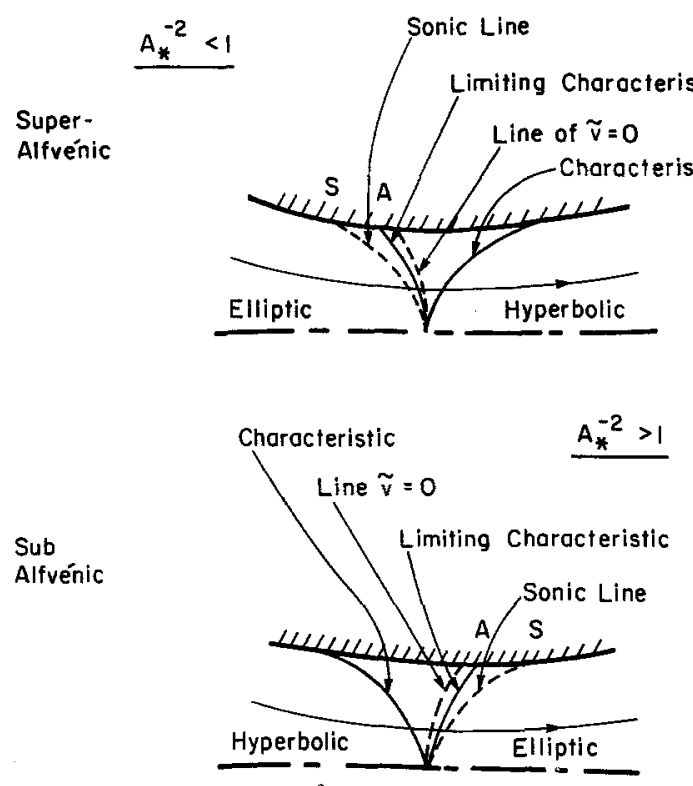

Figure 4. The structure of accelerating aligned field nozzle flow. 
The segment SA of the nozzle wall between the limiting characteristic and the sonic line can affect the flow in the upstream elliptic region when $A_{*}^{-2}<1$, even though the flow between the sonic line and the limiting characteristic is hyperbolic. When $A_{*}^{-2}>1$, the segment AS influences the downstream elliptic flow even though the flow between the limiting characteristic and the sonic line is in this case subsonic and hence hyperbolic. When $A_{*}^{-2}>1$, the structure of accelerating flow and the manner in which the boundaries influence the flow thus differ greatly from neutral gas flow. With $A_{*}^{-2}>1$ the Sears-Resler diagram (Fig. 1) shows that there is a low speed elliptic region upstream of the subsonic hyperbolic region in Fig. 4. Clearly this region must be taken into account in formulating the effect of upstream conditions upon the flow. However, the present paper is concerned only with the structure of the transonic region rather than with the overall flow.

\section{Taylor flow}

We first consider the limiting case $\alpha \rightarrow 0$. Then the solutions for $Z(S)$ become

$$
\left.\begin{array}{ll}
Z \rightarrow 2 S ; & S<0 \\
Z \rightarrow-S ; & S>0
\end{array}\right\} \text { Region A }
$$

In terms of physical variables the perturbation velocities corresponding to (36) are

$$
\begin{aligned}
& \left.\begin{array}{c}
\tilde{u}=4 x(\gamma+1)^{-\frac{1}{2}}+8\left(1-A_{*}^{-2}\right) y^{2} \\
\tilde{v}=16 x y+\frac{32}{3}(\gamma+1)^{\frac{1}{2}}\left(1-A_{*}^{-2}\right) y^{3}
\end{array}\right\} \frac{x}{(\gamma+1)^{\frac{1}{2}}\left(1-A_{*}^{-2}\right)}+y^{2}<0 \\
& \left.\begin{array}{l}
\tilde{u}=-2 x(\gamma+1)^{-\frac{1}{2}}+2\left(1-A_{*}^{-2}\right) y^{2} \\
\tilde{v}=4 x y-\frac{4}{3}(\gamma+1)^{\frac{1}{2}}\left(1-A_{*}^{-2}\right) y^{2}
\end{array}\right\} \frac{x}{(\gamma+1)^{\frac{1}{2}}\left(1-A_{*}^{-2}\right)}+y^{2}>0
\end{aligned}
$$

while the velocities corresponding to $(37)$ become

$$
\begin{aligned}
& \left.\begin{array}{l}
\tilde{u}=-2 x(\gamma+1)^{-\frac{1}{2}}+2\left(1-A_{*}^{-2}\right) y^{2} \\
\tilde{v}=4 x y-\frac{4}{3}(\gamma+1)^{\frac{1}{2}}\left(1-A_{*}^{-2}\right) y^{2}
\end{array}\right\} \frac{x}{(\gamma+1)^{\frac{1}{2}}\left(1-A_{*}^{-2}\right)}+y^{2}<0 \\
& \left.\begin{array}{l}
\tilde{u}=4 x(\gamma+1)^{-\frac{1}{2}}+8\left(1-A_{*}^{-2}\right) y^{2} \\
\tilde{v}=16 x y+\frac{32}{3}(\gamma+1)^{\frac{1}{2}}\left(1-A_{*}^{-2}\right) y^{3}
\end{array}\right\} \frac{x}{(\gamma+1)^{\frac{1}{2}}\left(1-A_{*}^{-2}\right)}+y^{2}>0
\end{aligned}
$$

Typical isovels corresponding to (38) are shown in Fig. 3(e) and 3(f). With $A_{*}^{-2}<1$ the flow behaves as in the neutral case with a pocket of supersonic flow near the wall; however, when $A_{*}^{-2}>1$ the overall flow becomes supersonic and there are pockets of subsonic flow near the nozzle walls. Such flows never occur in the neutral case. Velocity fields corresponding to (39) are shown in Fig. 3(i) and 3(j). With $A_{*}^{-2}<1$, the flow is again similar to the neutral case, being supersonic with only a single point at the center of the nozzle where the velocity reaches the sonic value. With $A_{*}^{-2}>1$, the flow is subsonic everywhere except for an isolated sonic point on the nozzle axis.

Physically it is more meaningful to separate the limiting solutions into subsonic and supersonic flows as in Fig. 5. The subsonic limiting solutions are shown in Fig. 5 (a) and 5(b), and the supersonic limiting solutions in $5(\mathrm{e})$ and $5(\mathrm{f})$. From Fig. 5 it is easy to visualize the change in the 


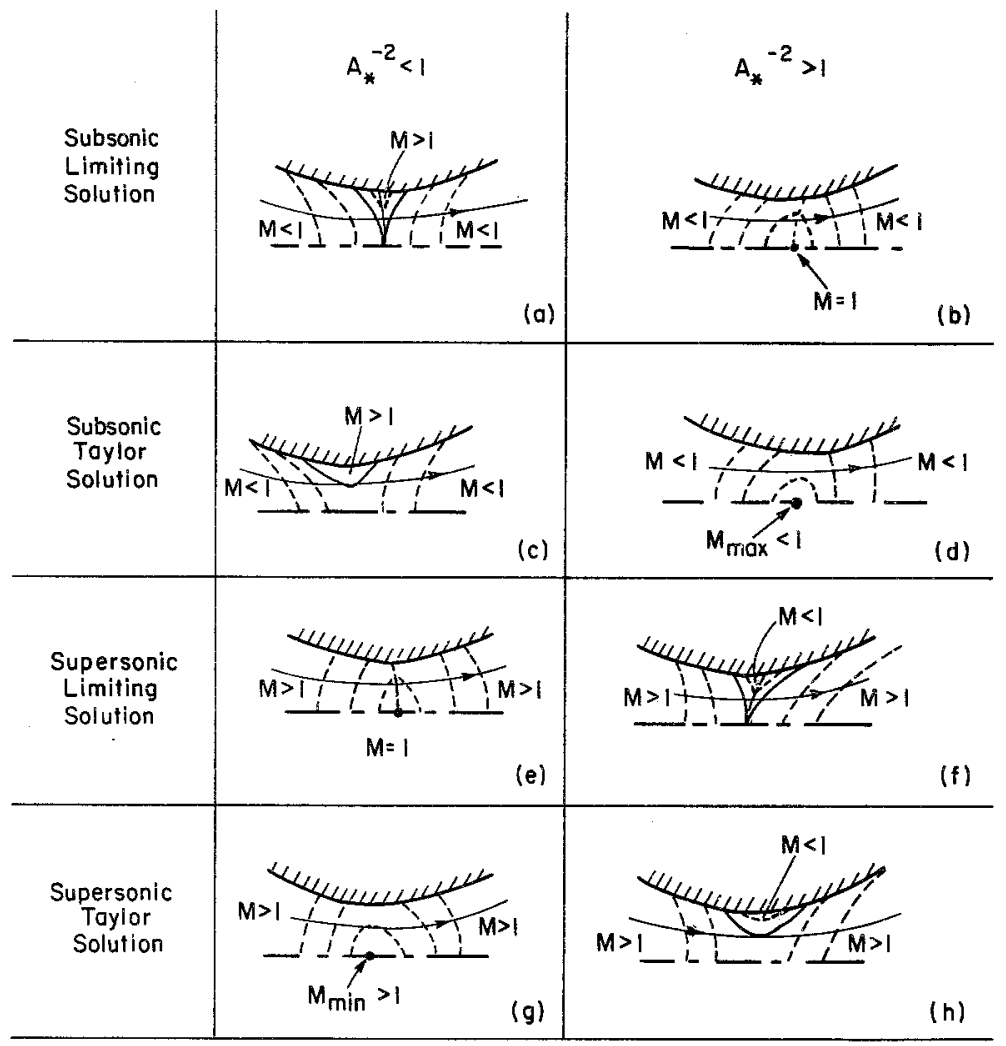

- Sonic Line

Figure 5. Subsonic and supersonic taylor type flow.

velocity field with increasing magnetic field (or increasing $A_{*}^{-2}$ ). As $A_{*}^{-2}$ increases the curvature of the isovels decreases and then reverses as $A_{*}^{-2}$ passes through the singular value of unity. In subsonic flow the supersonic pocket disappears and is replaced by an isolated sonic point as $A_{*}^{-2}$ increases beyond unity. The reverse transition with the formation of a subsonic pocket arises as $A_{*}^{-2}$ increases through unity in the supersonic case. The transformation (12) is singular when $A_{*}^{-2}=1$, consequently we have been unable to determine the precise nature of the transition between super and sub-Alfvénic flow. Higher order terms in the expansion (7) will probably be needed to establish the details of the flow when $A_{*}^{-2}=1$. It is interesting to observe that the subsonic flow with supersonic pockets when $A_{*}^{-2}<1$ is mirrored by the supersonic flow with subsonic pockets with $A_{*}^{-2}>1$. This mirroring is in accord with Tamada's observation [9] that the streamlines, shocks, and characteristics of a sub-Alfvenic flow are the same as those of a neutral gas flow with direction of the flow reversed.

In the non-limiting case $\alpha \neq 0$, the velocity perturbations $u$ and $v$ are given by

$$
\begin{aligned}
& \tilde{u}=2\left(1-A_{*}^{-2}\right)\left[Z(S)+2 y^{2}\right] \\
& \tilde{v}=4(\gamma+1)^{\frac{1}{2}}\left(1-A_{*}^{-2}\right)\left[y Z(S)+\frac{2 x y}{(\gamma+1)^{\frac{1}{2}}\left(1-A_{*}^{-2}\right)}+\frac{2}{3} y^{3}\right] \\
& S=\frac{x}{(\gamma+1)^{\frac{1}{2}}\left(1-A_{*}^{-2}\right)}+y^{2}
\end{aligned}
$$

The streamline of the flow are again defined by the equation

$$
\frac{d y}{d x}=\varepsilon^{2} \tilde{v}
$$




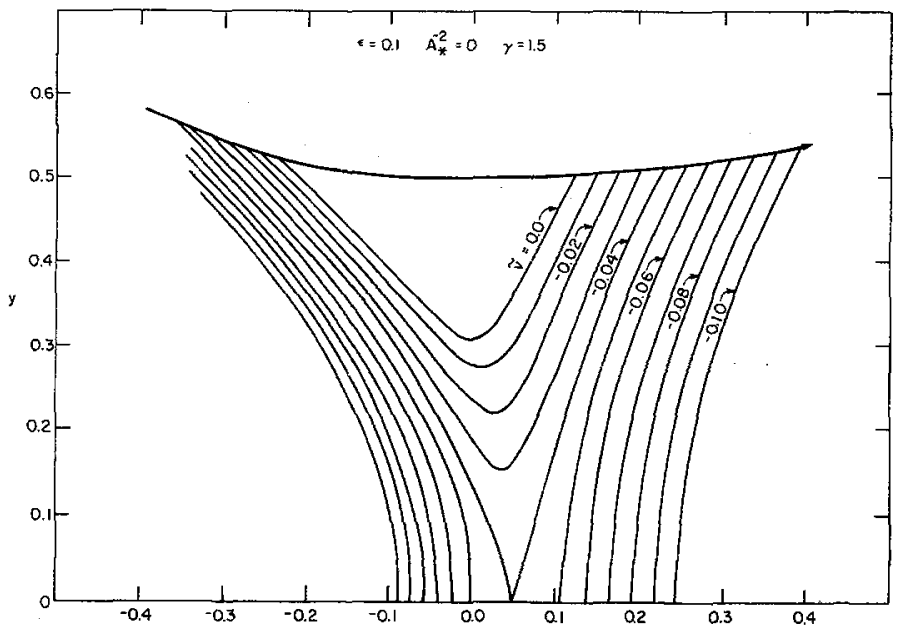

Figure 6. Subsonic symmetric flow $\left(A_{*}^{-2}<1\right)$.

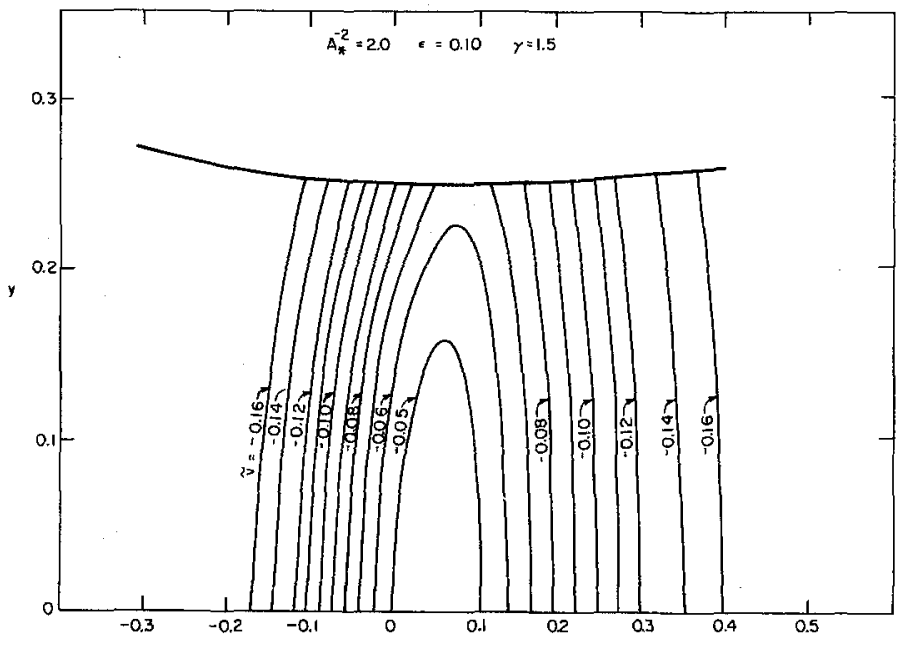

Figure 7. Subsonic symmetric flow $\left(A_{*}^{-2^{x}}>1\right)$.

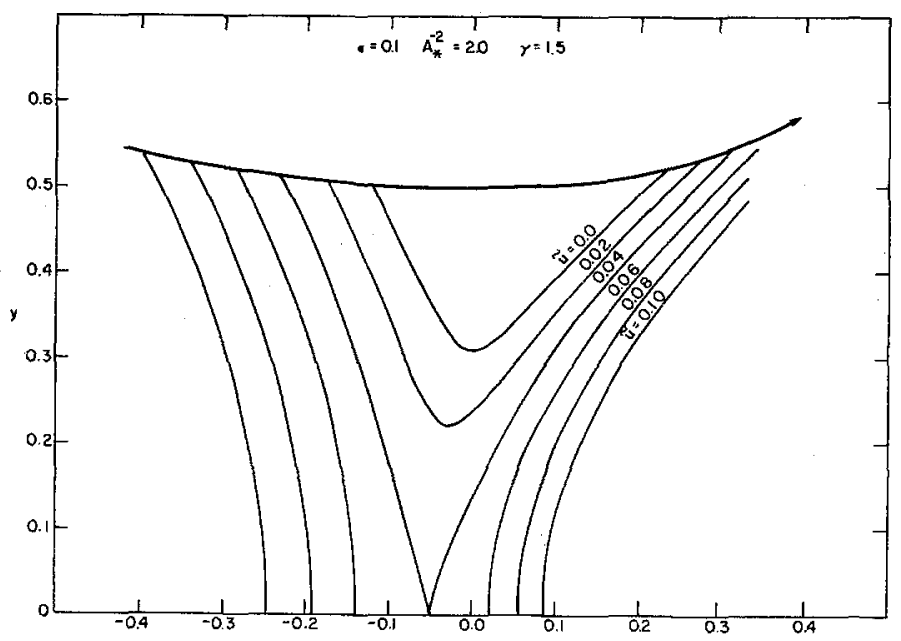

Figure 8. Supersonic symmetric flow $\left(A_{*}^{-2}>1\right)$. 
with the throat where $\tilde{v}=0$, taken at $y=1$. Typical nozzle flow patterns have been computed for $A_{*}^{-2}<1$ and $A_{*}^{-2}>1$ and are shown in Fig. 6, 7, and 8. It is, of course, characteristic of the similarity solution presented here that the streamlines cannot be specified a priori but will be different for each value of the integration constant $\alpha$ and for each value of the Alfvén number $A_{*}$. This in no way detracts from the utility of the similarity solution in displaying the structure of the flow near the throat.

Figure 6 shows subsonic symmetric flow of a neutral gas with $A_{*}^{-2}=0$. The flow is subsonic except for a supersonic pocket near the throat bounded by the isovel $u=0$, and is similar to that shown qualitatively in Fig. $3(\mathrm{~g})$ and 5 (c). A subsonic symmetric flow with $A_{*}^{-2}>1$, similar to Fig. $3(\mathrm{k})$, and $5(\mathrm{~d})$ is shown in Fig. 7. Here the region of maximum velocity occurs near the center of the nozzle rather than at the nozzle wall. Finally, Fig. 8 shows a supersonic symmetric flow with $A_{*}^{-2}>1$ similar to Fig. $3(\mathrm{~h})$ and $5(\mathrm{~h})$ and displays the strange phenomenon of a subsonic pocket adjacent to the nozzle wall. As in the limiting case the solutions for superAlfvénic flow (Fig. 6) and sub-Alfvénic flow (Fig. 8) mirror each other.

\section{Discussion}

We have used the Tomotika-Tamada [3] solution to investigate the structure of transonic aligned field MHD nozzle flow. As in neutral flow, many of the transonic phenomena observed within nozzles also arise in external flows.

No attempt has been made to test the stability of all the flow patterns displayed in Fig. 3 . In all likelihood the flows shown in Fig. 3(c), 3(i), and 3(k) will be unstable as they are very similar to the unstable neutral gas flows. Questions such as whether the presence of the magnetic field enhances or reduces stability, and what sub-Alfvénic flows are stable remain to be answered.

As indicated above, the Tomotika-Tamada [3] solution fails to describe the transition from Taylor-to Meyer flow, which is always accompanied by the formation of shock waves. The nature of this transitional process has been investigated by Watanabe [10] for aligned field flows.

Transonic flows such as those described above may arise in various plasma devices involving nozzle expansions or external flows of high density plasmas.

\section{Acknowledgement}

The authors are grateful for support of portions of this work by the U.S. Air Force under Contract AFOSR 72-2224, and by the U.S. Army Research Office in Durham, North Carolina under contract DAHC04-68-C-0008.

\section{REFERENCES}

[1] G. I. Taylor, Aeronaut. Res. Council Repts and Memoranda 1381, 1930; see also in The Scientific Papers of G.I. Taylor, (G. K. Batchelor, ed.), Vol. III, Cambridge Univ. Press London and New York 128-141.

[2] H. Grad, Reducible Problems in Magneto-Fluid Dynamic Steady Flows, Reviews of Modern Physics, 32 (1960) $830-847$.

[3] S. Tomotika and K. Tamada, Studies of Two-Dimensional Transonic Flows of a Compressible Fluid, Part I, Quart of Applied Math., VII, (1960) 381-397.

[4] T. Meyer, Über Zweidimensionale Bewegungsvorgänge in einen Gas, das mit Überschallgeschwindigkeit strömt, PH.D. dissertation, Göttingen, 1908. See also Foundation of Aerodynamics of High Speed (G. Carrier, ed.), Dover Publications, New York, (1951) $50-89$.

[5] M. Sichel, Two Dimensional Shock Structure in Transonic and Hypersonic Flow, Advances in Applied Mech., Academic Press Inc., New York, 11 (1971) 131-207.

[6] C. K. Chu, Magnetohydrodynamic Nozzle Flow with Three Transitions, The Phys. of Fluids, 5 (1960) 550-559.

[7] M. N. Kogan, On Magnetohydrodynamic Flows of Mixed Type, Prikl. Mat. i. Mekh. 25 (1961) 132-137; (translation in J. of Applied Math. and Mech, 25 (1961) 180-188.

[8] A. R. Seebass, Theory of Aligned Fields Magneto Gas Dynamic Flows, Ph.D. Thesis, Cornell University, Ithaca, New York (1963).

[9] K. Tamada, Two-Dimensional Transonic Flow of Perfectly Conducting Gas with Aligned Magnetic Field, Symposium Transsonicum, Springer-Verlag, Berlin, Göttingen, Heidelberg, (1964) 460-470.

[10] H. Watanabe, Aligned Fields Magnetogasdynamic Flow through Nozzles, Ph.D. Thesis, The University of Michigan, Ann Arbor, Michigan (1971). 